\title{
Fixed-Time Trajectory Tracking Control of Autonomous Surface Vehicle with Model Uncertainties and Disturbances
}

\author{
Jiawen Cui and Haibin Sun (iD \\ School of Engineering, Qufu Normal University, Rizhao 276826, China \\ Correspondence should be addressed to Haibin Sun; fengyun198212@163.com
}

Received 26 May 2020; Revised 23 September 2020; Accepted 29 September 2020; Published 15 October 2020

Academic Editor: Jianquan Lu

Copyright $\odot 2020$ Jiawen Cui and Haibin Sun. This is an open access article distributed under the Creative Commons Attribution License, which permits unrestricted use, distribution, and reproduction in any medium, provided the original work is properly cited.

\begin{abstract}
The issue of fixed-time trajectory tracking control for the autonomous surface vehicles (ASVs) system with model uncertainties and external disturbances is investigated in this paper. Particularly, convergence time does not depend on initial conditions. The major contributions include the following: (1) An integral sliding mode controller (ISMC) via integral sliding mode surface is first proposed, which can ensure that the system states can follow the desired trajectory within a fixed time. (2) Unknown external disturbances are absolutely estimated by means of designing a fixed-time disturbance observer (FTDO). By combining the FTDO and ISMC techniques, a new control scheme (FTDO-ISMC) is developed, which can achieve both disturbance compensation and chattering-free condition. (3) Aiming at reconstructing the unknown nonlinear dynamics and external disturbances, a fixed-time unknown observer (FTUO) is proposed, thus providing the FTUO-ISMC scheme that finally achieves trajectory tracking of ASVs with unknown parameters. Finally, simulation tests and detailed comparisons indicate the effectiveness of the proposed control scheme.
\end{abstract}

\section{Introduction}

With marine engineering operations developing and progressing, autonomous surface vehicles (ASVs) are instrumental in river and oil pipeline inspection, hull inspection, ocean survey, levee inspection, underwater archaeology, and underwater wreck inspection [1-5]. ASVs are usually perceived as a class of nonlinear dynamic systems equipped with complex external disturbances and model uncertainties [6]. It is an overwhelming matter to design a highly efficient controller for the ASV system.

Trajectory tracking is a basic problem for ASVs; however, as the system dynamics of ASV are highly nonlinear and there are unpredictable external disturbances in the marine environment, designing an effective controller for ASVs is a challenging issue. Many classical control algorithms such as feedback linearization [7], backstepping control [8], PID control [9], adaptive control [10], fuzzy control [11], and neural networks control [12] have been implemented to the trajectory tracking control of ASVs. The system states generally realize either asymptotic convergence or exponential convergence. In addition, many scholars have proposed many compound control methods in combination with above different control theories that apply to actual control systems [13-18], especially ASV systems [5, 6, 9], etc.

The finite-time control scheme profits from short divergence span and strong robustness, which is applied to various nonlinear control systems. In this respect, continuous finite-time control schemes for robotic manipulators have already been designed utilizing terminal sliding mode control theory in [19], and a nonsingular terminal sliding mode control scheme for a marine vehicle with complex unknowns has been presented in [20]. Moreover, a finitetime integral sliding controller in [21] has been designed to realize the path following of the ASVs.

Although the finite-time tracking control problems are achieved in the above references, the convergence time is to depend on the original states. When the initial time tends to infinity, the convergence time also tends to infinity. However, compared with the finite-time control schemes, the 
converge speed of the fixed-time control schemes is quite insensitive to initial condition [22, 23]. Recently, many fixed-time sliding mode control schemes have been proposed. For nonlinear systems with matched uncertainties and disturbances, a fixed-time nonsingular terminal sliding mode controller has been proposed in [24]. In [25], for the trajectory tracking control, a fixed-time nonsingular terminal sliding mode controller for a warship-launched submarine with multiple disturbances has been presented. In addition, a fixed-time sliding mode control for fault-tolerant trajectory tracking of an ASV has been designed in [26].

As known, chattering is an inherent phenomenon in sliding mode control. To reduce the chattering, a feasible solution is that disturbance observer-based control (DOBC) schemes are used to estimate external disturbances and model uncertainties and the disturbance estimation values are introduced into sliding mode control law. In order to improve the convergence speed and robustness, some finitetime disturbance observers were designed to estimate external disturbances and model uncertainties [27-32].

In this brief, the fixed-time trajectory tracking control scheme for ASVs with external disturbances and model uncertainties is explored. An integral sliding mode controller (ISMC) is firstly intended by using ISM surface for the ASV system without external disturbances, so that system states can attain the expected value in fixed time. Next, a fixed-time disturbance observer (FTDO) is designed to estimate the external disturbances and a new control scheme (FTDOISMC) is constructed to enable that the system states can accurately track the expected trajectory within fixed time even if there exist unexpected external disturbances. Furthermore, to guarantee good tracking performance against both disturbances and unknown system dynamics, a corresponding fixed-time unknown observer based on ISMC (FTUO-ISMC) control scheme is proposed. As a consequence, simulation results imply that the proposed control schemes can guarantee the system states to track the desired trajectory in a fixed time in spite of the ASV system subject to unknown disturbances and model uncertainties and the convergence speed is regardless of the origin states of the ASV system.

The remainder of this paper is structured as follows: In Section 2, some definitions and lemmas related to the trajectory tracking problem are formulated. The problem of the paper is described in Section 3. Section 4 describes the design of fixed-time controller and its stability analysis. Simulation results and discussion are mentioned in Section 5. And, Section 6 summarizes the main conclusions of this paper.

\section{Preliminaries}

Lemma 1 (see [33]). Consider the following double-integrator system:

$$
\begin{aligned}
\dot{x}_{1} & =x_{2}, \\
\dot{x}_{2} & =u, \\
x(0) & =x_{0},
\end{aligned}
$$

with the control law

$$
\begin{aligned}
u(t)= & -\left(k_{1}\left[x_{1}\right]^{\mathrm{e}_{1}}+k_{1}^{\prime}\left[x_{1}\right]+k_{2}^{\prime \prime}\left[x_{1}\right]^{\mathrm{Q}_{1 \prime} \prime}\right) \\
& -\left(k_{2}\left[x_{2}\right]^{\mathrm{Q}_{2}}+k_{2}^{\prime}\left[x_{2}\right]+k_{2}^{\prime \prime}\left[x_{2}\right]^{\mathrm{e}_{2} \prime}\right),
\end{aligned}
$$

where parameters $k_{i}>0, k_{i}^{\prime}>0, k_{i}^{\prime \prime}>0(i=1,2)$, and $\varrho_{i}, \varrho_{i}^{\prime}$, $(i=1,2)$ are chosen by

$$
\begin{aligned}
& \varrho_{1}=\frac{\varrho}{2-\varrho}, \\
& \varrho_{2}=\varrho, \\
& \varrho_{1}^{\prime}=\frac{4-3 \varrho}{2-\varrho}, \\
& \varrho_{2}^{\prime}=\frac{4-3 \varrho}{3-2 \varrho},
\end{aligned}
$$

with $\varrho \in(0,1)$ and $[x]^{\alpha}=|x|^{\alpha} \operatorname{sign}(x), \alpha \geq 0$. Next, the state of the double-integrator system is fixed-time stability with convergence time $t_{f}$.

Lemma 2 (see [34]). Consider the following nonlinear system:

$$
\begin{aligned}
\dot{x}(t) & =f(x, t), \\
f(0, t) & =0, \\
x(0) & =x_{0},
\end{aligned}
$$

where $x \in \mathbb{R}^{n}$ and $f: \mathbb{R}_{+} \times \mathbb{R}^{n} \longrightarrow \mathbb{R}^{n}$ is a nonlinear function. For the above system, suppose that there is a continuous radially unbounded function $V: \mathbb{R}^{n} \longrightarrow \mathbb{R}_{+} \mathbb{U}\{0\}$ which satisfies

(1) $V(x)=0$, when $x=0$

(2) $\dot{V}(x) \leq-\alpha V^{p}(x)-\beta V^{q}(x)$ for some $\alpha, \beta, p, q>0$, with $0<p<1$ and $q>1$

The considered nonlinear system is globally fixed-time stable within the settling time T satisfying

$$
T \leq T_{\max }:=\frac{1}{\alpha(1-p)}+\frac{1}{\beta(q-1)} .
$$

\section{System Modeling and Problem Formulation}

The kinematics and dynamics of the ASV system regarded as rigid body with three degrees of freedom (3-DOF) are represented by [6]

$$
\begin{gathered}
\dot{\eta}=R(\varphi) v, \\
M \dot{v}=-C(v) v-D(v) v-g(\eta)+\tau+M R^{T} d_{l}(t),
\end{gathered}
$$

where $\eta=[x, y, \varphi]^{T}$ is the location $(x, y)$ and course angle $(\varphi)$ of ASVs in an earth-fixed inertial frame, $v=[u, v, r]^{T}$ is the linear velocities $(u, v)$ and angular rate $(r)$ in the bodyfixed frame, $\tau=\left[F_{u}, F_{v}, F_{r}\right]^{T}$ stands for the actual control thrust, and $d_{l}(t)$ denotes unsuspected external disturbances owing to complex surface environment including wind, 
waves, and ocean current. The rotation matrix $R(\varphi)$ is defined by

$$
R(\varphi)=\left[\begin{array}{ccc}
\cos \varphi & -\sin \varphi & 0 \\
\sin \varphi & \cos \varphi & 0 \\
0 & 0 & 1
\end{array}\right],
$$

being provided with the following characters: $R^{T}(\varphi)$ $R(\varphi)=I, \quad \dot{R}(\varphi)=R(\varphi) S(r), \quad \forall \varphi \subseteq[0,2 \pi]$, and $R^{T}(\varphi) S(r)$ $R(\varphi)=R(\varphi) S(r) R^{T}(\varphi)=S(r)$, where

$$
S(r)=\left[\begin{array}{ccc}
0 & -r & 0 \\
r & 0 & 0 \\
0 & 0 & 0
\end{array}\right]
$$

is the inertia matrix $M=M^{T}>0$ and

$$
M=\left[\begin{array}{ccc}
m-X_{\dot{u}} & 0 & 0 \\
0 & m-Y_{\dot{v}} & m x_{g}-Y_{\dot{r}} \\
0 & m x_{g}-N_{\dot{v}} & I_{z}-N_{\dot{r}}
\end{array}\right],
$$

where $m$ is the mass of the system, $I_{z}$ is the inertia matrix concerned with the yaw angle, $Y_{\dot{r}}=N_{\dot{\gamma}}$, and $X_{*}, Y_{*}$, and $Z_{*}$ denote the corresponding hydrodynamic derivatives. Coriolis and centripetal matrix $C(v)=-C(v)^{T}$ have the following form:

$$
C(v)=\left[\begin{array}{ccc}
0 & 0 & c_{13}(v) \\
0 & 0 & c_{23}(v) \\
-c_{13}(v) & -c_{23}(v) & 0
\end{array}\right],
$$

and the damping matrix $D(v)$ is described by

$$
D(v)=\left[\begin{array}{ccc}
d_{11}(v) & 0 & 0 \\
0 & d_{22}(v) & d_{23}(v) \\
0 & d_{32}(v) & d_{33}(v)
\end{array}\right],
$$

where $\quad c_{13}(v)=-m\left(x_{g} r+v\right)+X_{\dot{u}} v+Y_{\dot{r}} r, \quad c_{23}(v)=m u-$ $X_{u} u, \quad$ and $\quad d_{11}(v)=-X_{u}-X_{|u| u}|u|-X_{u u u} u u^{2}, \quad d_{22}(v)=$ $-Y_{v}-Y_{|v| v}|v|, \quad d_{23}(v)=-Y_{r}-Y_{|v| r \mid}|v|-Y_{|r| r}|r|, \quad d_{32}(v)=$ $-N_{\nu}-N_{|v| v}|\nu|-N_{|r| v}|r|$, and $\quad d_{33}(v)=-N_{r}-N_{|\nu| r}|\nu|-$ $N_{|r| r}|r|$. And $g(\eta)$ is the gravity and buoyancy forces and moments, which is usually used as a constant in ASV.

Consider the desired trajectory as follows:

$$
\begin{aligned}
\dot{\eta}_{d} & =R\left(\varphi_{d}\right) v_{d}, \\
M \dot{v}_{d} & =-C\left(v_{d}\right) v_{d}-D\left(v_{d}\right) v_{d}+\tau_{d},
\end{aligned}
$$

where $\eta_{d}=\left[x_{d}, y_{d}, \varphi_{d}\right]^{T}$ and $v_{d}=\left[u_{d}, v_{d}, r_{d}\right]^{T}$ denote its desired position and velocity vectors, and the model contains no unknown nonlinear dynamics including external disturbances and model uncertainties.

Assumption 1. For disturbance vector $d_{l}(t)$, it is given that constants $\kappa_{i}$ satisfies $\left|\dot{d}_{l i}(t)\right| \leq \kappa_{i}$, where $\kappa_{i}$ is an unknown nonnegative bounded constant.

The control purpose, in this paper, is to design fixed-time trajectory tracking control schemes so that the practical position and velocity (6)-(7) can precisely pursuit the expected ones (13)-(14), respectively.

\section{Controller Design and Stability Analysis}

4.1. Coordinate Transformation. Consider coordinate transformations as follows:

$$
\begin{aligned}
& \sigma=R v, \\
& \sigma \in\left\{\sigma, \sigma_{d}\right\}, \\
& v \in\left\{v, v_{d}\right\}, \\
& R \in\left\{R, R_{d}\right\},
\end{aligned}
$$

where $\sigma=\left[\sigma_{1}, \sigma_{2}, \sigma_{3}\right]^{T}, \sigma_{d}=\left[\sigma_{d 1}, \sigma_{d 2}, \sigma_{d 3}\right]^{T}, R=R(\varphi)$, and $R_{d}=R\left(\varphi_{d}\right)$.

By combining (6)-(7) and (15), we can obtain

$$
\begin{aligned}
& \dot{\eta}=\sigma, \\
& \dot{\sigma}=R M^{-1} \tau+\Theta(\eta, \sigma)+d_{l}(t),
\end{aligned}
$$

where

$$
\begin{aligned}
\Theta(\eta, \sigma)= & S(\sigma) \sigma-R M^{-1}\left(C\left(R^{T} \sigma\right)+D\left(R^{T} \sigma\right)\right) R^{T} \\
& \sigma-R M^{-1} g(\eta) .
\end{aligned}
$$

Similarly, together with (13), (14), and (15), we obtain

$$
\begin{aligned}
& \dot{\eta}_{d}=\sigma_{d}, \\
& \dot{\sigma}_{d}=R_{d} M^{-1} \tau_{d}+\Theta_{d}\left(\eta_{d}, \sigma_{d}\right),
\end{aligned}
$$

where

$$
\begin{aligned}
\Theta_{d}\left(\eta_{d}, \sigma_{d}\right)= & S\left(\sigma_{d}\right) \sigma_{d}-R_{d} M^{-1}\left(C\left(R_{d}^{T} \sigma_{d}\right)\right. \\
& \left.+D\left(R_{d}^{T} \sigma_{d}\right)\right) R_{d}^{T} \sigma_{d} .
\end{aligned}
$$

Define the position and velocity error $\eta_{e}=\left[\eta_{e 1}\right.$, $\left.\eta_{e 2}, \eta_{e 3}\right]^{T}$ and $\sigma_{e}=\left[\sigma_{e 1}, \sigma_{e 2}, \sigma_{e 3}\right]^{T}$. Then, we have

$$
\begin{aligned}
& \dot{\eta}_{e}=\sigma_{e}, \\
& \dot{\sigma}_{e}=R M^{-1} \tau-R_{d} M^{-1} \tau_{d}+d_{l}(t)+\Theta_{e}\left(\eta, \sigma, \eta_{d}, \sigma_{d}\right),
\end{aligned}
$$

where

$$
\Theta_{e}\left(\eta, \sigma, \eta_{d}, \sigma_{d}\right)=\Theta(\eta, \sigma)-\Theta_{d}\left(\eta_{d}, \sigma_{d}\right) .
$$

4.2. Design of the ISMC without External Disturbances. In this section, an ISMC is firstly proposed for tracking error systems (20) and (21) without external disturbances, and the fixed-time stability is verified.

The ISM manifold is designed as follows:

$$
s\left(\sigma_{e}(t)\right)=\sigma_{e}(t)+\int_{t_{0}}^{t} u_{n}\left(\sigma_{e}(\theta)\right) \mathrm{d} \theta,
$$

where 


$$
u_{n}=k_{1}\left[\eta_{e}\right]^{\varrho_{1}}+k_{1}^{\prime}\left[\eta_{e}\right]+k_{1}^{\prime \prime}\left[\eta_{e}\right]^{e_{1}^{\prime}}+k_{2}\left[\sigma_{e}\right]^{\varrho_{2}}+k_{2}^{\prime}\left[\sigma_{e}\right]+k_{2}^{\prime \prime}\left[\sigma_{e}\right]^{e_{2}^{\prime}} .
$$

and parameters are provided in Lemma 1.

The ISMC can be designed as

$$
\tau_{\text {ISMC }}=M R^{-1}\left(R_{d} M^{-1} \tau_{d}-\Theta_{e}-u_{n}-\xi_{1}[s]^{\alpha}-\xi_{2}[s]^{\beta}\right),
$$

where $0<\alpha<1, \beta>1, \xi_{1}>0$, and $\xi_{2}>0$.

Theorem 1 (ISMC). Consider tracking error systems (20)(21) without external disturbances, and an ISMC is designed by (24). Systems (6) and (7) can converge to desired trajectories (13) and (14) within a fixed time, i.e., $\eta \equiv \eta_{d}$ and $v \equiv v_{d}$, when $t>T_{f 1}$.

Proof. There are two processes in the whole verification: the reaching and the sliding phases:

(i) Step 1: taking the derivative of ISM surfaces (22) and (23) along error systems (20) and (21) without external disturbances $d_{l}(t)$ and combining with the control law (24), we can obtain

$$
\dot{s}=\dot{\sigma}_{e}+u_{n}=-\xi_{1}[s]^{\alpha}-\xi_{2}[s]^{\beta} .
$$

Take the candidate Lyapunov function as follows:

$$
V(s)=\frac{1}{2} s^{2}
$$

Differentiating it along the dynamics (25), we obtain

$$
\begin{aligned}
\dot{V}(s) & =s \dot{s}=s\left(-\xi_{1}[s]^{\alpha}-\xi_{2}[s]^{\beta}\right)=-\xi_{1}|s|^{\alpha+1}-\xi_{2}|s|^{\beta+1} \\
& =-2^{\alpha+1 / 2} \xi_{1} V^{\alpha+1 / 2}-2^{\beta+1 / 2} \xi_{2} V^{\beta+1} .
\end{aligned}
$$

According to Lemma 2, it is claimed that the ISM control law will let the system states reach the ISM surface $s=0$ within a fixed time.

(ii) Step 2: at that moment, error system (20) will reduce to the following system:

$$
\begin{aligned}
& \dot{\eta}_{e}=\sigma_{e}, \\
& \dot{\sigma}_{e}=-u_{n}\left(\eta_{e}, \sigma_{e}\right) .
\end{aligned}
$$

By applying Lemma 1, we have that system (28) is globally fixed-time stable. Eventually, errors $\eta_{e}$ and $\sigma_{e}$ are converging to zero within a fixed time $t_{f}$, so this completes the proof. Under the ISMC scheme, the convergence time of the ASV system is $T_{f 1}=T_{0}+t_{f}$, where $T_{0}=1 / 2^{(\alpha-1) / 2}$ $\xi_{1}(1-\alpha)+1 / 2^{(\beta-1) / 2} \xi_{2}(\beta-1)$.

Remark 1. External disturbances are not considered in ISMC excogitation, but they are actually exist in the actual environment. Therefore, controller design for the ASV system with external disturbances is essential.

4.3. Design of FTDO-ISMC. To achieve accurate tracking performance, in this section, a FTDO is built to estimate the external disturbances. Inspired by [35-37], a FTDO algorithm is established as

$$
\begin{aligned}
\chi_{0} & =\sigma-\chi, \\
\dot{\chi} & =R M^{-1} \tau-R_{d} M^{-1} \tau_{d}+\Theta_{e}\left(\eta, \sigma, \eta_{d}, \sigma_{d}\right)+\widehat{d}_{l}(t)+\psi_{i}, \\
\psi_{i} & =\lambda_{1 i}\left|\chi_{0 i}\right|^{1 / 2} \operatorname{sign}\left(\chi_{0 i}\right)+\lambda_{2 i}\left|\chi_{0 i}\right|^{\gamma_{i}} \operatorname{sign}\left(\chi_{0 i}\right), \\
\dot{\vec{d}}_{l}(t) & =\beta_{i} \operatorname{sign}\left(\chi_{0 i}\right), \quad i=1,2,3,
\end{aligned}
$$

where $\chi_{0}=\left[\chi_{01}, \chi_{02}, \chi_{03}\right]^{T}$ is an auxiliary variable, $\psi=\left[\psi_{1}\right.$, $\left.\psi_{2}, \psi_{3}\right]^{T}, \widehat{d}_{l}(t)=\left[\widehat{d}_{l 1}(t), \widehat{d}_{l 2}(t), \widehat{d}_{l 3}(t)\right]^{T}$ denotes the estimation of $d_{l}(t), \lambda_{1 i}$ and $\lambda_{2 i}$ are constants greater than zero, $\beta_{i}>\kappa_{i}$, and $\gamma_{i}>1$.

The error dynamic of the observer is described as

$$
\begin{aligned}
& \dot{\chi}_{0 i}=\chi_{1 i}-\lambda_{1 i}\left|\chi_{0 i}\right|^{1 / 2} \operatorname{sign}\left(\chi_{0 i}\right)-\lambda_{2 i}\left|\chi_{0 i}\right|^{\gamma_{i}} \operatorname{sign}\left(\chi_{0 i}\right), \\
& \dot{\chi}_{1 i}=-\beta_{i} \operatorname{sign}\left(\chi_{0 i}\right)+\dot{d}_{l i}(t),
\end{aligned}
$$

where $\chi_{1 i}=d_{l i}(t)-\widehat{d}_{l i}(t)$ is the estimation error of external disturbance. It can be derived from $[36,37]$ that observer error system (18) is fixed-time stable according to Assumption 1, i.e., when $t>t_{1}, \chi_{0 i}=\chi_{1 i}=0$. And the $t_{1}$ satisfies $t_{1} \leq\left(1 / \lambda_{2}(\gamma-1) \varepsilon^{\gamma-1}+2 \varepsilon^{1 / 2} / \lambda_{1}\right)\left(1+1 / m\left((1 / M)-\left(h\left(\lambda_{1}\right) /\right.\right.\right.$ $\left.\left.\lambda_{1}\right)\right)$ with $\varepsilon>0, \quad M=\alpha-\kappa, m=\alpha+\kappa$, and $h\left(\lambda_{1}\right)=$ $1 / \lambda_{1}+\left(2 \exp (1) / m \lambda_{1}\right)^{1 / 3}$.

Theorem 2 (FTDO-ISMC). Consider tracking error systems (20)-(21) with external disturbances satisfying Assumption 1; then, a FTDO-ISMC is designed as

$\tau_{\mathrm{FTDO}-\mathrm{ISMC}}=M R^{-1}\left(R_{d} M^{-1} \tau_{d}-\Theta_{e}-u_{n}-\widehat{d}_{l}(t)-\xi_{1}[s]^{\alpha}-\xi_{2}[s]^{\beta}\right)$,

with $\widehat{d}_{l}$ estimated by FTDO (30). Systems (6) and (7) can converge to desired trajectories (13) and (14) within a fixed time, i.e., $\widehat{d}_{l}(t) \equiv d_{l}(t), \eta \equiv \eta_{d}$, and $v \equiv v_{d}$, when $t>T_{f 2}$.

Proof. The derivative of ISM surface (13) is rewritten as

$$
\dot{s}=\chi_{1}-\xi_{1}[s]^{\alpha}-\xi_{2}[s]^{\beta} \text {. }
$$

And derivative Lyapunov function $V$ based on (32) obtains

$$
\dot{V}=s \chi_{1}-\xi_{1}|s|^{\alpha+1}-\xi_{2}|s|^{\beta+1}
$$

When the time $t>t_{1}, \widehat{d}_{l}(t)$ equals $d_{l}(t)$, that is to say that the disturbance estimation error $\chi_{1 i}$ converges to zero within a fixed time. (33) is rewritten as

$$
\dot{V}=-\xi_{1}|s|^{\alpha+1}-\xi_{2}|s|^{\beta+1} .
$$

Based on Lemma 2, (34) is described by $\dot{V}=-2^{\alpha+1 / 2} \xi_{1} V^{\alpha+1 / 2}-2^{\beta+1 / 2} \xi_{2} V^{\beta+1 / 2}$ and we obtain that the 
ISM manifold $s=0$ will be arrived within a fixed time $T_{1}=1 / 2^{(\alpha-1) / 2} \xi_{1}(1-\alpha)+1 / 2^{(\beta-1) / 2} \xi_{2}(\beta-1)$. The system is still the same as (28) while $s=0$. In other words, the tracking error system states $\eta_{e}$ and $v_{e}$ are still sufficient to ensure convergence along the sliding surface in a fixed time $t_{f}$. Under the FTDO-ISMC scheme, the convergence time of the ASV system is $T_{f 2} \leq t_{1}+T_{1}+t_{f}$. This concludes the proof.

Remark 2. Note that we do not testify that the proposed controller (31) can ensure the boundedness of errors $\eta_{e}$ and $\sigma_{e}$ in the time quantum $\left[0, t_{1}\right]$ since the analysis of the dynamics of tracking error system is a difficult subject due to the complex nonlinear terms. In view of this reason, we have done a large number of simulations for ASV systems (6)-(7) under observer (29) and control laws (31), in which any divergence phenomenon is not observed. Practically, for the purpose of guaranteeing the boundedness of error system states in engineering, a bounded control rule can be applied in $\left[0, t_{1}\right]$. Therefore, it can assume that all states will not be divergent in fixed time in advance of the observer error dynamics converging.

Remark 3. Notice that it is difficult for $\Theta_{e}$ to obtain the real value because $C(v)$ and $D(v)$ are unknown because of existing uncertainties in ASV uncertainties. The FTDO only estimates the external disturbances and the influence of nonlinear terms is not considered, which will be solved in this context.

4.4. Design of FTUO-ISMC. In this section, the unknown nonlinear term consisting of $C(v), D(v)$, and $g(\eta)$ together with the external disturbances $d_{l}(t)$ are regarded as the lumped disturbances. Therefore, rewriting tracking error systems (20) and (21) yields

$$
\begin{aligned}
& \dot{\eta}_{e}=\sigma_{e}, \\
& \dot{\sigma}_{e}=R M^{-1} \tau-R_{d} M^{-1} \tau_{d}-\Theta_{d}+S(\sigma) \sigma+\Phi,
\end{aligned}
$$

where

$$
\Phi=-R M^{-1}\left(C\left(R^{T} \sigma\right)+D\left(R^{T} \sigma\right)\right) R^{T} \sigma-R M^{-1} g(\eta)+d_{l}(t),
$$

is an unknown nonlinearity regarded as the lumped disturbances. According to [29], an assumption is presented as follows.

Assumption 2. There exists a bounded constant $h_{0}$ such that $|\dot{\Phi}| \leq h_{0}$.

A FTUO is presented as

$$
\begin{aligned}
e_{0} & =\sigma-e, \\
\dot{e} & =R M^{-1} \tau-R_{d} M^{-1} \tau_{d}-\Theta_{d}\left(\eta_{d}, \sigma_{d}\right)+S(\sigma) \sigma+\widehat{\Phi}+\psi_{i}, \\
\psi_{i} & =\lambda_{1 i}\left|e_{0 i}\right|^{1 / 2} \operatorname{sign}\left(e_{0 i}\right)+\lambda_{2 i}\left|e_{0 i}\right|^{\gamma_{i}} \operatorname{sign}\left(e_{0 i}\right), \\
\dot{\hat{\Phi}}_{i} & =\beta_{i} \operatorname{sign}\left(e_{0 i}\right), \quad i=1,2,3,
\end{aligned}
$$

where $e_{0}=\left[e_{01}, e_{02}, e_{03}\right]^{T}$ is an auxiliary variable, $\psi=\left[\psi_{1}, \psi_{2}, \psi_{3}\right]^{T}, \widehat{\Phi}=\left[\widehat{\Phi}_{1}, \widehat{\Phi}_{2}, \widehat{\Phi}_{3}\right]^{T}$ is the estimation of the unknown lumped disturbances $\Phi, \lambda_{1 i}$ and $\lambda_{2 i}$ are positive constants, $\beta_{i}>h_{0}$, and $\gamma_{i}>1$.

The error dynamic of the observer is described as

$$
\begin{aligned}
& \dot{e}_{0 i}=e_{1 i}-\lambda_{1 i}\left|e_{0 i}\right|^{1 / 2} \operatorname{sign}\left(e_{0 i}\right)-\lambda_{2 i}\left|e_{0 i}\right|^{\gamma_{i}} \operatorname{sign}\left(e_{0 i}\right), \\
& \dot{e}_{1 i}=-\beta_{i} \operatorname{sign}\left(e_{0 i}\right)+\dot{\Phi}_{i}(t),
\end{aligned}
$$

where the lumped disturbance estimation error is described as $e_{1 i}=\Phi_{i}-\widehat{\Phi}_{i}$. With the help of Assumption 2, observer error system (38) is found to be fixed-time stable according to [36, 37], i.e., when $t>t_{2}, e_{0 i}=e_{1 i}=0$. And, the $t_{2} \leq$ $\left(\left(1 / \lambda_{2}(\gamma-1) \varepsilon^{\gamma-1}\right)+\left(2 \varepsilon^{1 / 2} / \lambda_{1}\right)\right)\left(1+\left(1 / m\left(1 / M-h\left(\lambda_{1}\right) /\right.\right.\right.$ $\left.\left.\lambda_{1}\right)\right)$ ) with $M=\alpha-h_{0}$ and $m=\alpha+h_{0}$ is different from the FTDO.

Theorem 3 (FTUO-ISMC). Considering tracking error systems (35)-(36) with the unknown lumped disturbances satisfying Assumption 2, a FTUO-ISMC is designed as

$$
\tau_{\text {FTUO-ISMC }}=M R^{-1}\left(R_{d} M^{-1} \tau_{d}+\Theta_{d}-S(\sigma) \sigma\right),
$$

with $\widehat{\Phi}$ estimated by FTUO (40). Systems (6) and (7) can converge to desired ones (13) and (14) within a fixed time, i.e., $\widehat{\Phi} \equiv \Phi, \eta \equiv \eta_{d}$, and $v \equiv v_{d}$, when $t>T_{f 3}$.

Proof. The derivative of ISM surface (22) is redescribed as follows:

$$
\dot{s}=e_{1}-\xi_{1}[s]^{\alpha}-\xi_{2}[s]^{\beta} .
$$

And the derivation of Lyapunov function (25) will be changed as

$$
\dot{V}=s e_{1}-\xi_{1}|s|^{\alpha+1}-\xi_{2}|s|^{\beta+1} .
$$

When the time $t>t_{2}, \widehat{\Phi}$ equals $\Phi$; to put it differently, the unknown lumped disturbance estimation error $e_{1}$ converges to zero in a fixed time, which makes equation (41) become (27) such that system states arrive at $s=0$ within a fixed time $T_{2}=\left(1 / 2^{(\alpha-1) / 2}\right) \xi_{1}(1-\alpha)+\left(1 / 2^{(\beta-1) / 2}\right) \xi_{2}(\beta-1)$ based on Lemma 2. The tracking error system is the same as (28) after reaching the sliding surface, and it converges to origin along the sliding surface in a fixed time based on Lemma 1. Under the FTUO-ISMC scheme, the convergence time of the ASV system is $T_{f 3} \leq t_{2}+T_{2}+t_{f}$. The proof is absolutely accomplished.

Remark 4. Although the states of tracking error systems (35)-(36) under control law (40) are not proved to be bounded within $\left[0, t_{2}\right]$ analogous to Remark 2, sufficient simulation results have been shown that any state does not diverge in $\left[0, t_{2}\right]$. Hence, we still suppose that the states of (35) are bounded within the time period $\left[0, t_{2}\right]$.

Remark 5. The ISMC scheme is designed based on integrated sliding mode surface for ASV systems without external disturbances. The FTDO-ISMC scheme is designed when there are external disturbances in the ASV system. The 
FTUO-ISMC scheme is designed considering the model uncertainties and external disturbances of the ASV system. The similarity lies in that ISMC, FTDO-ISMC, and FTUOISMC schemes are all based on the state feedback to design the integral sliding mode controllers.

\section{Simulation}

For the sake of illustrating the efficiency and superiority of the developed ISMC, FTDO-ISMC, and FTUO-ISMC points at trajectory following of an ASV, simulation machine adopted the surface vehicle CyberShip II [38] whose main parameters can be found in [27].

In this section, simulation proposed three control laws verifying that the system states can track the trajectory given by (13) and (14) within a fixed time, where desired control input $\tau_{d}$ is conducted by $\tau_{d}=\left[5,2 \cos ^{2}(0.1 \pi t)\right.$, $\left.\sin ^{2}(0.1 \pi t)\right]^{T}$. The origin condition is given by $\eta(0)=$ $[2,1, \pi / 2]^{T}, \quad v(0)=[0,0,0]^{T}, \quad \eta_{d}(0)=[1,2, \pi / 4]^{T}, \quad$ and $v_{d}(0)=[0,0,0]^{T}$.

5.1. Simulation on the ISMC. The ISMC scheme is simulated without considering external disturbances, and the parameters of this scheme are chosen as follows: $\varrho=0.7$, $k_{i}=k_{i}^{\prime}=k_{i}^{\prime \prime}=5, \xi_{1}=\xi_{2}=3, \alpha=0.5$, and $\beta=2$. It is clearly expressed from Figures 1-3 that the desired system state is fully tracked by the system states within a fixed time.

In Figures 4 and 5, curves of tracking error are shown under two sets of initial conditions. The case one is as above and the case two is $\eta(0)=[1,1, \pi / 3]^{T}, v(0)=[1,0,0]^{T}$, $\eta_{d}(0)=[1,0, \pi / 2]^{T}$, and $v_{d}(0)=[0,1,0]^{T}$. The convergence time can be demonstrated to be similar in distinct initial states. The control input of ISMC is shown in Figure 6.

5.2. Simulation on the FTDO-ISMC. In general, the external disturbances always exist in the ASV dynamics. The unknown external disturbances $d_{l}(t)$ are chosen as

$$
d_{l}(t)=\left[\begin{array}{c}
9 \sin \left(0.1 \pi t-\frac{\pi}{5}\right) \\
6 \sin \left(0.3 \pi t+\frac{\pi}{6}\right) \\
3 \sin \left(0.2 \pi t+\frac{\pi}{3}\right)
\end{array}\right] .
$$

And the parameters of the proposed FTDO and corresponding controller $\tau_{\text {FTDO-ISMC }}$ are chosen as follows:

$\lambda_{1 i}=\lambda_{2 i}=6, \quad \beta_{1}=9, \quad \beta_{2}=6, \quad \beta_{3}=3 ; \quad \gamma_{1}=\gamma_{2}=0.3$, $\gamma_{3}=0.8 ; \varrho=0.7, k_{i}=k_{i}^{\prime}=k_{i}^{\prime \prime}=5, \xi_{1}=\xi_{2}=4, \alpha=0.5$, and $\beta=2$.

Accordingly, simulation results of FTDO-ISMC scheme are illustrated in Figures 7-13. From Figures 7-12, compared to the performance with ISMC, the FTDO-ISMC scheme can also accurately control for tracking control of the ASV system among brief time in the presence of external disturbances. As can be seen from Figure 12, the FTDO can accurately estimate the external disturbances. The control

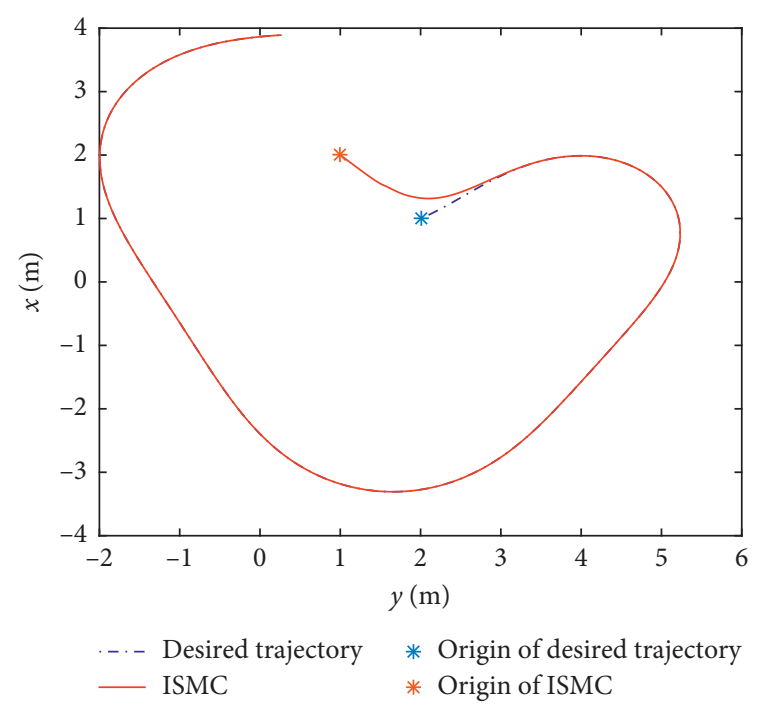

FIGURE 1: Planar trajectory tracking on ISMC.
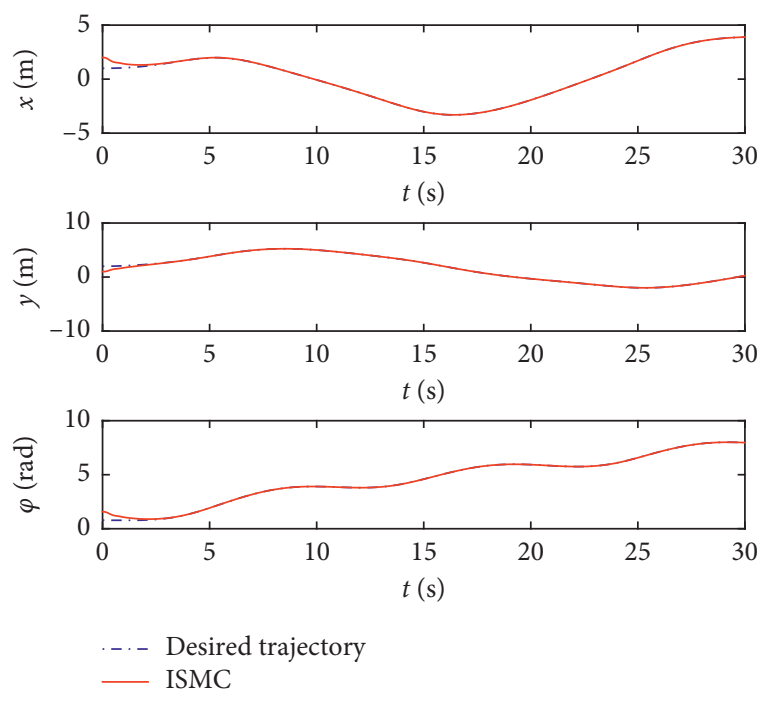

Figure 2: Position tracking on ISMC.
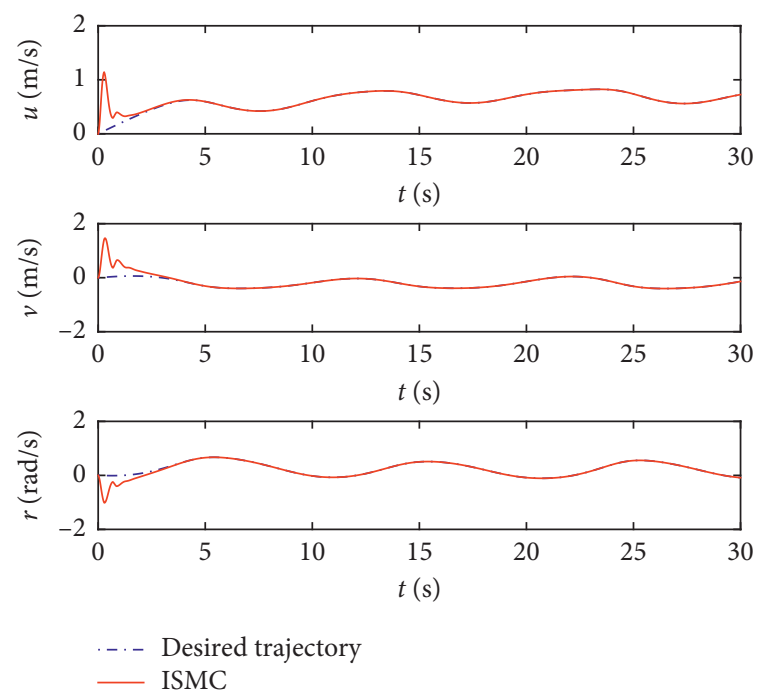

FIGURE 3: Velocity tracking on ISMC. 

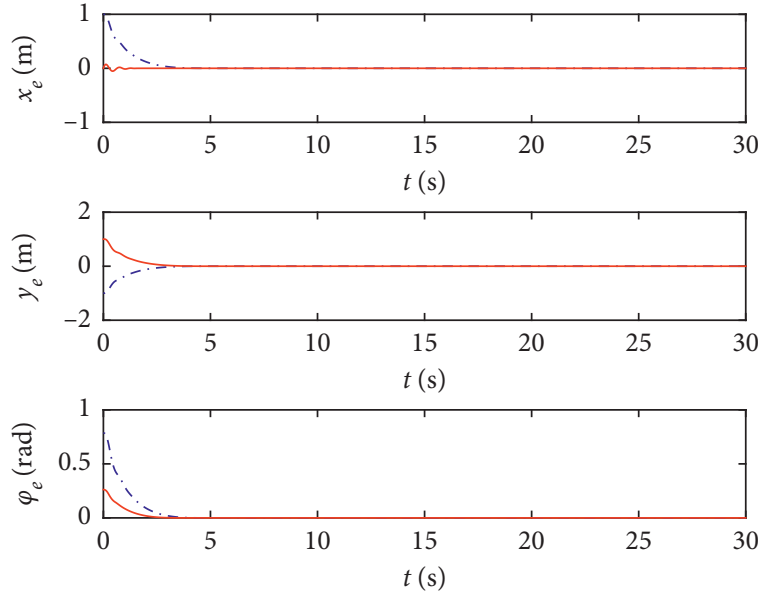

-. - Case one of ISMC

- Case two of ISMC

FIgURE 4: Position tracking errors on ISMC with different conditions.
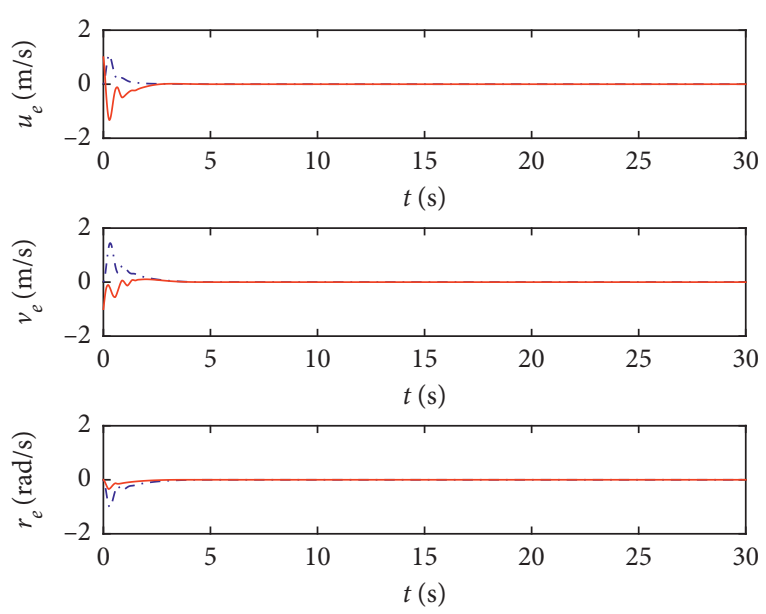

- - - Case one of ISMC

_ Case two of ISMC

FIgURE 5: Velocity tracking errors on ISMC with different conditions.
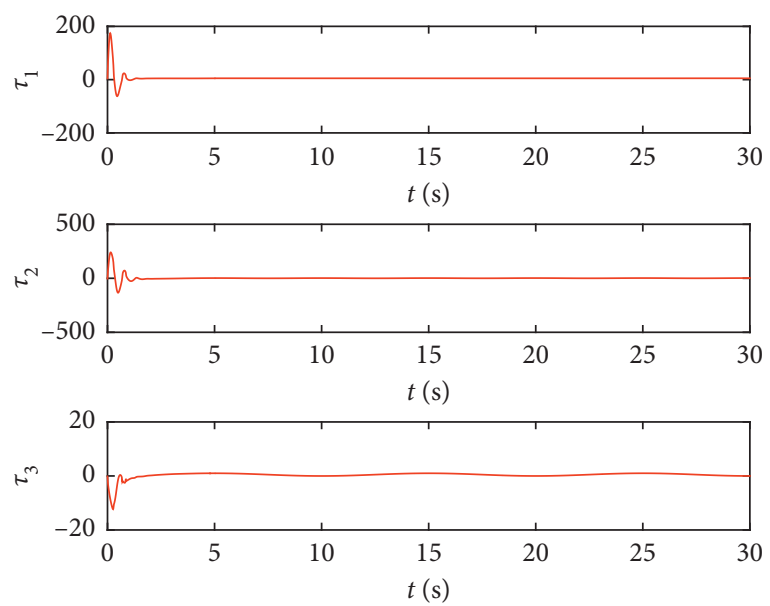

Figure 6: Control input on ISMC.

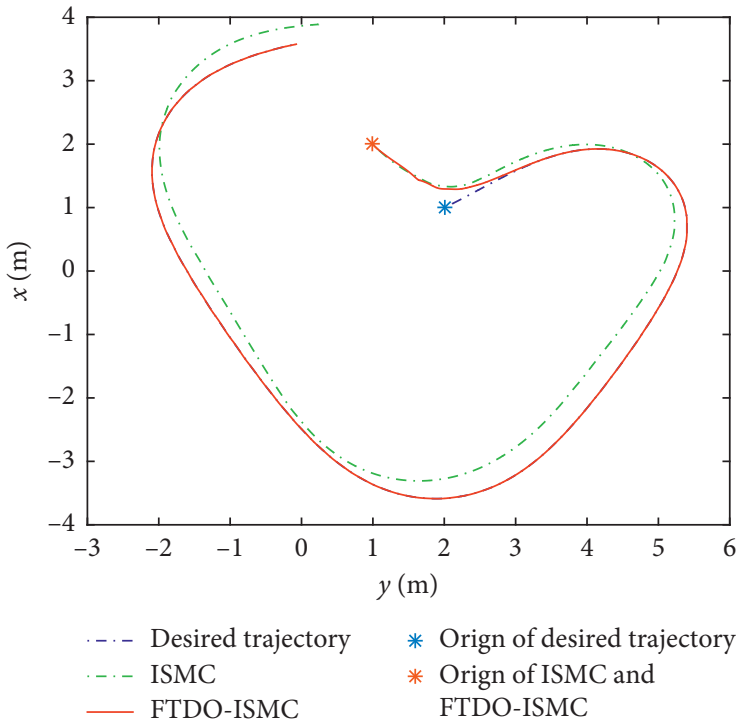

FIgURE 7: Planar trajectory tracking on ISMC and FTDO-ISMC.
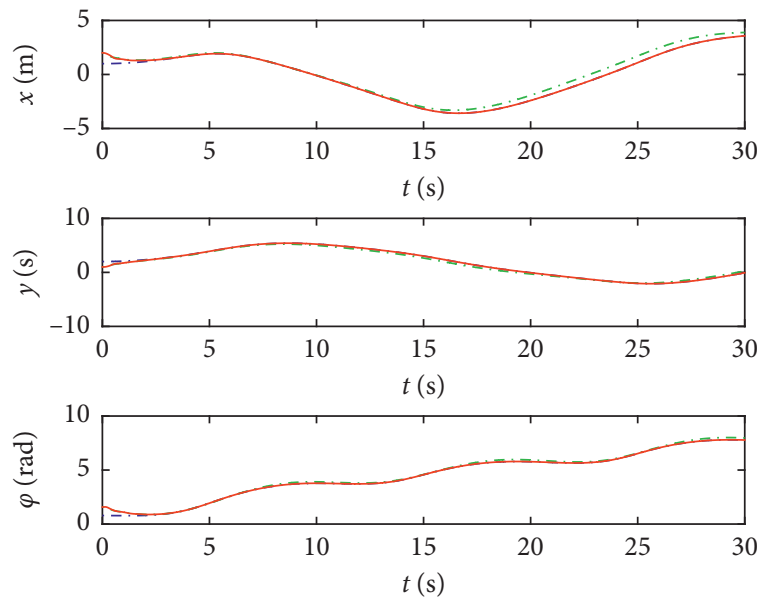

-.- Desired trajectory

-. - ISMC

- FTDO-ISMC

FIGURE 8: Position tracking on ISMC and FTDO-ISMC.
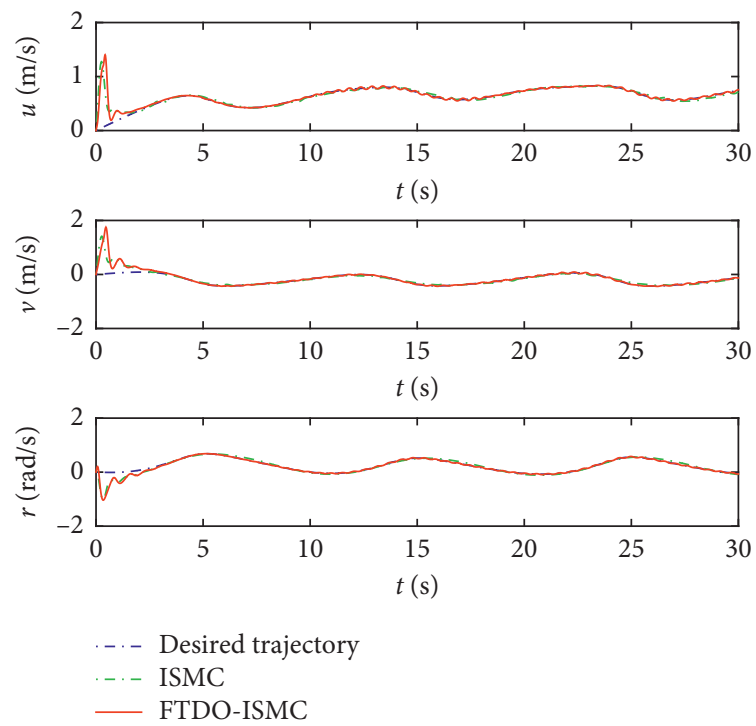

FIGURE 9: Velocity tracking on ISMC and FTDO-ISMC. 

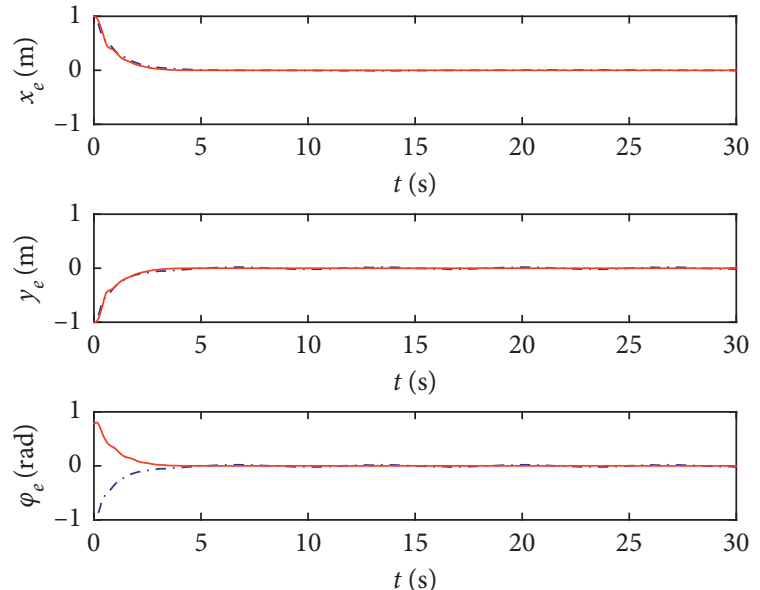

-.. ISMC

— FTDO-ISMC

FIgURE 10: Position tracking errors on ISMC and FTDO-ISMC.
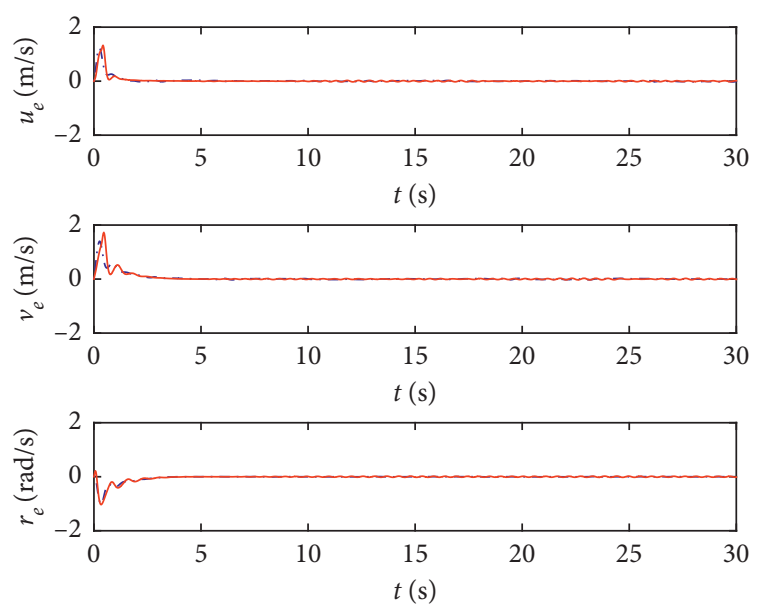

-.. ISMC

— FTDO-ISMC

FIgURE 11: Velocity tracking errors on ISMC and FTDO-ISMC.
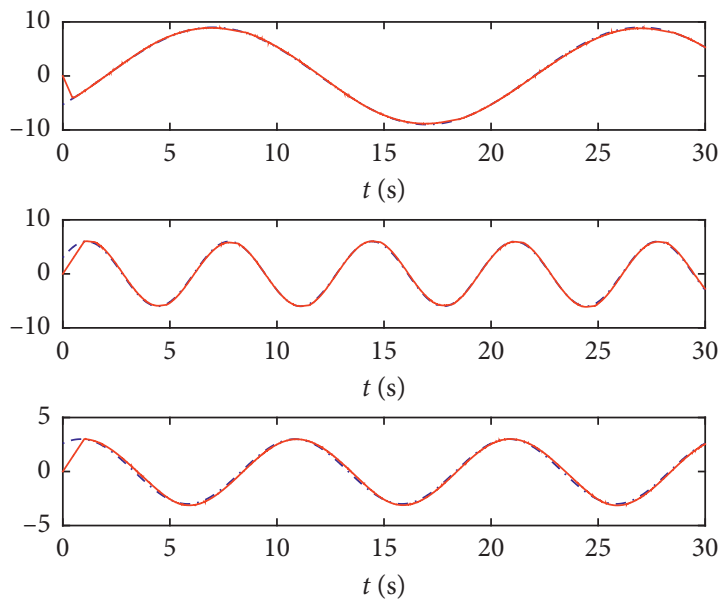

$-\cdot-d_{1}(t)$

Estimation of $d_{1}(t)$

FIGURE 12: External disturbances and their estimations on FTDOISMC.
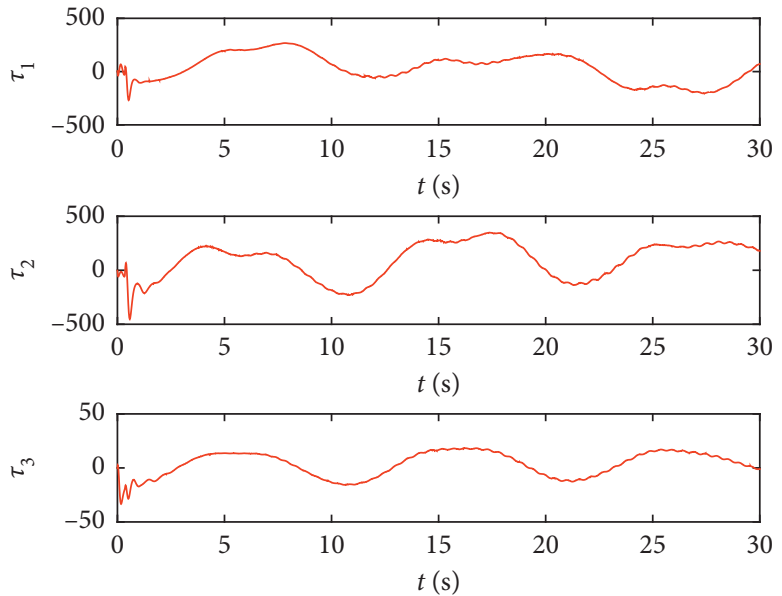

FIgURE 13: Control input under FTDO-ISMC.

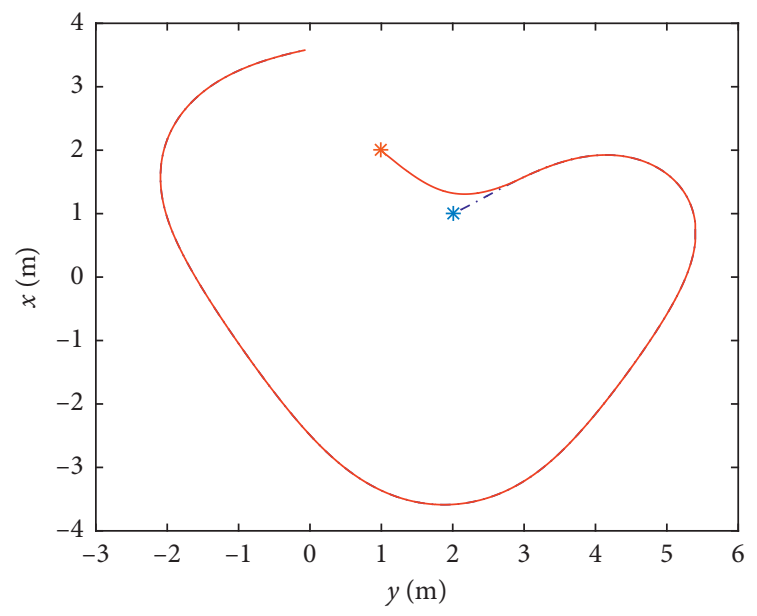

- . - Desired trajectory $\quad *$ Origin of desired trajectory — FTUO-ISMC * Origin of FTUO-ISMC

Figure 14: Planar trajectory tracking.
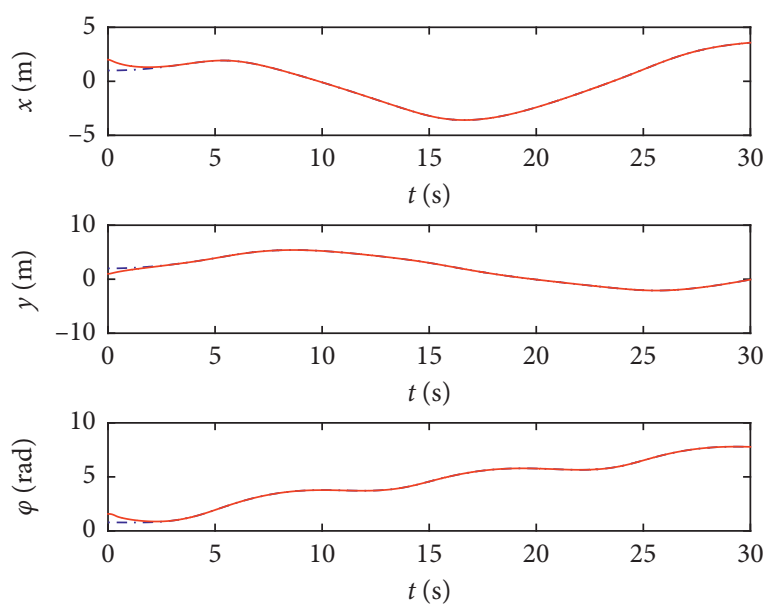

- - Desired trajectory

- FTUO-ISMC

Figure 15: Position tracking on FTUO-ISMC. 

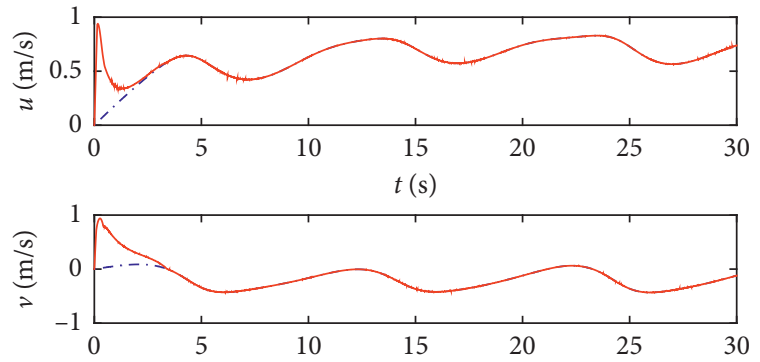

$t(\mathrm{~s})$

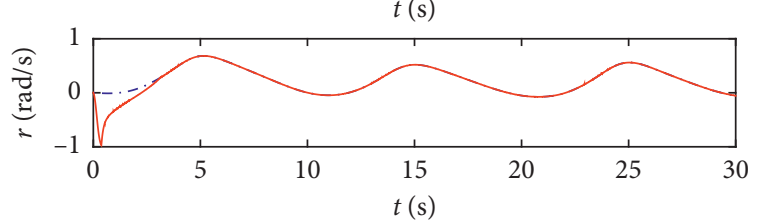

-. - Desired trajectory

- FTUO-ISMC

FIgURE 16: Velocity tracking on FTUO-ISMC.
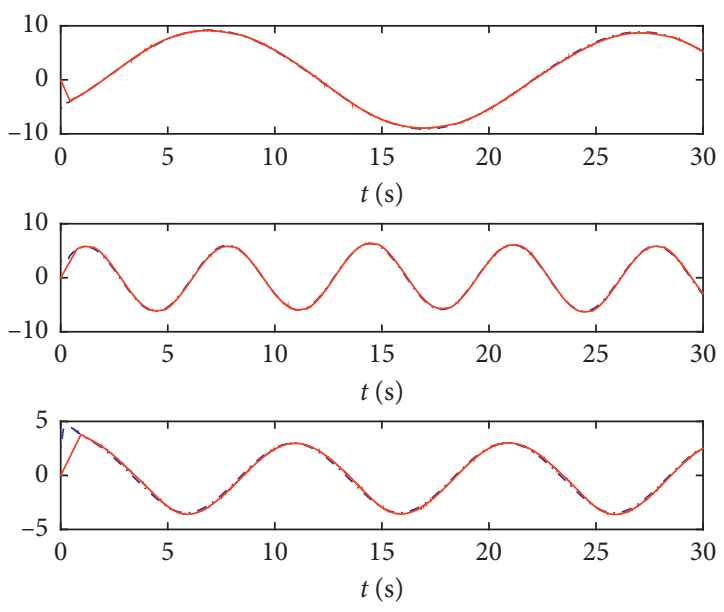

... Unknown disturbance

_ Unknown disturbance estimation

FIGURE 17: Unknown disturbance and its estimation on FTUOISMC.

input of the ASV system under FTDO-ISMC scheme is shown in Figure 13. Therefore, it can be concluded that the FTDO-ISMC scheme can achieve good tracking performance while external disturbances are existing.

5.3. Simulation on the FTUO-ISMC. The unknown lumped disturbances composed of the external disturbances and the model uncertainties of the ASV system are estimated together by the FTUO. The parameters of the proposed FTUO and corresponding controller $\tau_{\text {FTUO-ISMC }}$ are chosen as follows: $\lambda_{1 i}=\lambda_{2 i}=6, \beta_{1}=9, \beta_{2}=7$, and $\beta_{3}=4 ; \gamma_{1}=\gamma_{2}=$ 0.3 and $\gamma_{3}=0.6 ; \varrho=0.3, k_{i}=k_{i}^{\prime}=k_{i}^{\prime \prime}=5, \xi_{1}=4, \xi_{2}=4$, $\alpha=0.1$, and $\beta=2$.

The simulation results of the ASV system under FTUOISMC are all provided in Figures 14-17. It could be described from Figures 14-16 that the vehicle can follow the expected trajectory accurately with the fixed time while the unknown lumped disturbances are presenting. In Figure 17, curves of unknown lumped disturbances and estimations are presented. From this figure, we can see that the proposed FTUO can effectively estimate the lumped disturbances. As a conclusion, the tracking performance of the FTUO-ISMC is satisfied for the ASV system with external disturbances and parameter uncertainties.

\section{Conclusion}

In this paper, the problem of fixed-time trajectory tracking control has been investigated for ASVs with external disturbances and model uncertainties. By introducing integral sliding mode surface, an ISMC scheme has been proposed for ASVs, which can achieve position and velocity tracking in fixed time. Then, a fixed-time disturbance observer (FTDO) has been designed to estimate the external disturbances. The control scheme (FTDO-ISMC) proposed by combining the ISM surface and the output of FTDO can accurately track the desired trajectory for ASVs with the external disturbances. A fixed-time unknown observer (FTUO) has been developed to calculate the unknown lumped disturbances, and a control law in the light of fixed-time unknown observer (FTUOISMC) has been proposed to achieve accurate disturbances attenuation and trajectory tracking for the ASVs. Results on simulation have been used to illustrate the superiority and efficiency of the control schemes proposed.

\section{Data Availability}

No data were used to support this study.

\section{Conflicts of Interest}

The authors declares that there are no conflicts of interest.

\section{Acknowledgments}

This work was partially supported by the National Natural Science Foundation of China under Grants 61773236, $61773235,61703233,61873331$, and 61803225 , partially by the Taishan Scholar Project of Shandong Province under Grants TSQN20161033 and ts201712040, partially by the Natural Science Foundation of Anhui Province (19080805MF219), partially by the Postdoctoral Science Foundation of China (2017M612236 and 2019T120574), and partially by the Interdisciplinary Scientific Research Projects of Qufu Normal University under Grant (xkjjc201905).

\section{References}

[1] A. P. Aguiar and J. P. Hespanha, "Trajectory-tracking and path-following of underactuated autonomous vehicles with parametric modeling uncertainty," IEEE Transactions on Automatic Control, vol. 52, no. 8, pp. 1362-1379, 2007.

[2] Z. Zeng, L. Lian, K. Sammut, F. He, Y. Tang, and A. Lammas, "A survey on path planning for persistent autonomy of autonomous underwater vehicles," Ocean Engineering, vol. 110, no. Part A, pp. 303-313, 2015. 
[3] X. Xiang, C. Yu, and Q. Zhang, "On intelligent risk analysis and critical decision of underwater robotic vehicle," Ocean Engineering, vol. 140, pp. 453-465, 2017.

[4] L. Perera, P. Oliveira, and C. Soares, "Maritime traffic monitoring based on vessel detection, tracking, state estimation, and trajectory prediction," IEEE Transactions on Intelligent Transportation Systems, vol. 13, no. 3, pp. 1188-1200, 2012.

[5] A. Behal, D. M. Dawson, W. E. Dixon, and Y. Fang, "Tracking and regulation control of an underactuated surface vessel with nonintegrable dynamics," IEEE Transactions on Automatic Control, vol. 47, no. 3, pp. 495-500, 2002.

[6] T. Fossen, Marine Control Systems: Guidance, Navigation, and Control of Ships, Rigs and Underwater VehiclesMarine Cybernetics AS, Trondheim, Norway, 2002.

[7] L.-x. Pan, H.-z. Jin, and L.-l. Wang, "Robust control based on feedback linearization for roll stabilizing of autonomous underwater vehicle under wave disturbances," China Ocean Engineering, vol. 25, no. 2, pp. 251-263, 2011.

[8] C. Shen, Y. Shi, and B. Buckham, "Trajectory tracking control of an autonomous underwater vehicle using lyapunov-based model predictive control," IEEE Transactions on Industrial Electronics, vol. 65, no. 7, pp. 5796-5805, 2018.

[9] X. Xiang, C. Yu, and Q. Zhang, "Robust fuzzy 3D path following for autonomous underwater vehicle subject to uncertainties," Computers \& Operations Research, vol. 84, pp. 165-177, 2017.

[10] J.-H. Li and P.-M. Lee, "Design of an adaptive nonlinear controller for depth control of an autonomous underwater vehicle," Ocean Engineering, vol. 32, no. 17-18, pp. 2165-2181, 2005.

[11] J. Guo, F.-C. Chiu, and C.-C. Huang, "Design of a sliding mode fuzzy controller for the guidance and control of an autonomous underwater vehicle," Ocean Engineering, vol. 30, no. 16, pp. 2137-2155, 2003.

[12] M.-j. Zhang and Z.-z. Chu, "Adaptive sliding mode control based on local recurrent neural networks for underwater robot," Ocean Engineering, vol. 45, pp. 56-62, 2012.

[13] C. Huang, J. Lu, D. W. Ho, G. Zhai, and J. Cao, "Stabilization of probabilistic boolean networks via pinning control strategy," Information Sciences, vol. 510, pp. 205-217, 2020.

[14] C. Huang, X. Zhang, H. K. Lam, and S. H. Tsai, "Synchronization analysis for nonlinear complex networks with reaction-diffusion terms using fuzzy-model-based approach," IEEE Transactions on Fuzzy Systems, p. 1. In press, 2020.

[15] C. Huang, J. Lu, G. Zhai, J. Cao, G. Lu, and M. Perc, "Stability and stabilization in probability of probabilistic boolean networks," IEEE Transactions on Neural Networks and Learning Systems, pp. 1-11. In press, 2020.

[16] P. Liu, L. Li, K. Shi, and J. Lu, "Pinning stabilization of probabilistic boolean networks with time delays," IEEE Access, vol. 8, pp. 154050-154059, 2020.

[17] X. Ji, J. Lu, J. Lou, J. Qiu, and K. Shi, “A unified criterion for global exponential stability of quaternion-valued neural networks with hybrid impulses," International Journal of Robust and Nonlinear Control, 2020, In press.

[18] X. Li, X. Yang, and T. Huang, "Persistence of delayed cooperative models: impulsive control method," Applied Mathematics and Computation, vol. 342, pp. 130-146, 2019.

[19] C. Yu, X. Yu, B. Shirnzadeh, and Z. Man, "Continuous finitetime control for robotic manipulators with terminal sliding mode," Automatica, vol. 44, no. 11, pp. 1957-1964, 2005.

[20] N. Wang, S. Lv, W. Zhang, Z. Liu, and M. J. Er, "Finite-time observer based accurate tracking control of a marine vehicle with complex unknowns," Ocean Engineering, vol. 145, pp. 406-415, 2017.
[21] S. Yu and X. Long, "Finite-time consensus for second-order multi-agent systems with disturbances by integral sliding mode," Automatica, vol. 54, pp. 158-165, 2015.

[22] J. Hu, G. Sui, X. Lv, and X. Li, "Fixed-time control of delayed neural networks with impulsive perturbations," Nonlinear Analysis: Modelling and Control, vol. 23, no. 6, p. 904, 2018.

[23] D. Yang, X. Li, and J. Qiu, "Output tracking control of delayed switched systems via state-dependent switching and dynamic output feedback," Nonlinear Analysis: Hybrid Systems, vol. 32, pp. 294-305, 2019.

[24] L. Zhang, Y. Wang, Y. Hou, and H. Li, "Fixed-time sliding mode control for uncertain robot manipulators," IEEE Access, vol. 7, pp. 149750-149763, 2019.

[25] Z. Zuo, "Non-singular fixed-time terminal sliding mode control of non-linear systems," IET Control Theory \& Applications, vol. 9, no. 4, pp. 545-552, 2015.

[26] L. Zhang, C. Wei, L. Jing, and N. Cui, "Fixed-time sliding mode attitude tracking control for a submarine-launched missile with multiple disturbances," Nonlinear Dynamics, vol. 93, no. 4, pp. 2543-2563, 2018.

[27] N. Wang, X. Pan, and S. Su, "Finite-time fault-tolerant trajectory tracking control of an autonomous surface vehicle," Journal of the Franklin Institute, vol. 357, no. 16, pp. 11114-11135, 2019.

[28] N. Wang, C. Qian, J.-C. Sun, and Y.-C. Liu, "Adaptive robust finite-time trajectory tracking control of fully actuated marine surface vehicles," IEEE Transactions on Control Systems Technology, vol. 24, no. 4, pp. 1454-1462, 2016.

[29] X. Fang, F. Liu, and S. Zhao, "Trajectory tracking control for manned submersible system with disturbances via disturbance characterization index approach," International Journal of Robust and Nonlinear Control, vol. 29, no. 16, pp. 5641-5653, 2019.

[30] J. Ni, L. Liu, M. Chen, and C. Liu, "Fixed-time disturbance observer design for brunovsky systems," IEEE Transactions on Circuits and Systems. II-Express Briefs, vol. 65, no. 3, pp. 341-345, 2018.

[31] J. Sun, J. Yi, Z. Pu, and X. Tan, "Fixed-time sliding mode disturbance observer-based nonsmooth backstepping control for hypersonic vehicles," IEEE Transactions on Systems, Man, and Cybernetics: Systems, vol. 2, pp. 1-10. In press, 2018.

[32] J. Zhang, S. Yu, and Y. Yan, "Fixed-time extended state observer-based trajectory tracking and point stabilization control for marine surface vessels with uncertainties and disturbances," Ocean Engineering, vol. 186, p. 106109, 2019.

[33] B. Tian, Z. Zuo, X. Yan, and H. Wang, "A fixed-time output feedback control scheme for double integrator systems," Automatica, vol. 80, pp. 17-24, 2017.

[34] A. Polyakov, "Nonlinear feedback design for fixed-time stabilization of linear control systems," IEEE Transactions on Automatic Control, vol. 57, no. 8, pp. 2106-2110, 2012.

[35] H. Sun, L. Hou, G. Zong, and X. Yu, "Fixed-time attitude tracking control for spacecraft with input quantization," IEEE Transactions on Aerospace and Electronic Systems, vol. 55, no. 1, pp. 124-134, 2019.

[36] M. Basin, C. Bharath Panathula, and Y. Shtessel, "Multivariable continuous fixed-time second-order sliding mode control: design and convergence time estimatio," IET Control Theory \& Applications, vol. 11, no. 8, pp. 1104-1111, 2017.

[37] M. Basin, C. B. Panathula, and Y. Shtessel, "Adaptive uniform finite-/fixed-time convergent second-order sliding-mode control," International Journal of Control, vol. 89, no. 9, pp. 1777-1787, 2016.

[38] R. Skjetne, T. I. Fossen, and P. V. Kokotović, "Adaptive maneuvering, with experiments, for a model ship in a marine control laboratory," Automatica, vol. 41, no. 2, pp. 289-298, 2005. 